\title{
A microfluidic method for investigating ion-specific bubble coalescence in salt solutions
}

\author{
Jianlong Wang, ${ }^{\mathrm{a}}$ Say Hwa Tan, ${ }^{\mathrm{b}}$ Anh V. Nguyen, ${ }^{\mathrm{a}}$ Geoffrey M. Evans, ${ }^{\mathrm{c}}$ and Nam-Trung Nguyen* ${ }^{\mathrm{b}}$ \\ a. School of Chemical Engineering, University of Queensland, Brisbane, QLD 4072, Australia \\ b. Queensland Micro- and Nanotechnology Centre, Griffith University, Brisbane, QLD 4111, Australia. \\ c. School of Engineering, University of Newcastle, Callaghan, NSW 2308, Australia.
}

\begin{abstract}
This paper reports the direct and precise information on critical bubble coalescence in salt solutions using microfluidics. We directly visualised the bubble coalescence in a micro-channel using highspeed imaging and evaluated the bubble coalescence time to determine the critical coalescence concentration of sodium halide solutions. At minimum bubble coalescence time, the critical salt concentrations are ion-specific which is similar to the Jones-Ray effect on the minimum of surface tension of salt solutions. We found the capacity of sodium halide salts to inhibit bubble coalescence follows the order of $\mathrm{NaF}>\mathrm{NaCl}>\mathrm{NaBr}>\mathrm{NaI}$. The novel tool reported here eliminates the effect of inertial forces on the coalescence time and overcomes the inherent uncertainties in conventional largescale devices and methods.
\end{abstract}

\section{INTRODUCTION}

Bubble coalescence in different salt solutions governs many interesting but unexpected phenomena. These processes are found in nature and industrial applications. Examples include the different effects of breaking waves of winds on the foaminess of seawater and fresh water in the lake. Industrially, purification of mined coal from clays using air bubbles is possible in seawater and other saline waters. Fine bubbles were often generated without the use of conventional surfactants. ${ }^{1}$ Unlike surfactants, salts often increase surface tension promoting bubble coalescence. Ironically, both surface-active compound (surfactants) and surface-inactive compound (inorganic salts) inhibit bubble coalescence. More interestingly, these compounds can only inhibit bubble coalescence above a critical concentration, referred to as the critical coalescence concentration (CCC). 2, 3, 4, 5, 6, 7, 8, 9, 10

Despite the inconsistent definitions of CCC in the currently available literature, different techniques have been developed to determine the CCC. ${ }^{2}$ Briefly, the techniques can be categorised into: (i) the bubble swarm experiments; (ii) the bubble pair method and (iii) the film balance technique. All these techniques have been not satisfactory due to their inherent shortcomings and uncertainties. Consequently, significant discrepancies in the CCC values existed, when results obtained via different techniques were compared. ${ }^{2}$ 
In the bubble swarm experiments, the population of bubbles in a swarm is studied and the CCC is defined either as the concentration corresponding to $50 \%$ bubble coalescence ${ }^{11}$ or as the concentration above which a constant bubble size is reached. ${ }^{6,12}$ The bubble swarm experiment is useful for studying the effects of flow conditions on bubble coalescence. However, the measurements of CCC highly depend on hydrodynamic conditions such as turbulence. ${ }^{13}$ Therefore, the uncertainties of the measurement are significant by nature. For example, the CCC of $\mathrm{NaCl}$ ranges from $0.31 \mathrm{M}$ to 0.778 $\mathrm{M}$ via the measurement of the size distribution in a bubble column. ${ }^{2}$ In addition, obtaining the average bubble size by measuring the whole bubble population is time-consuming.

In the bubble pair method, two bubbles are generated from adjacent capillaries placed either side by side or facing each other. The CCC is now defined as the concentration at which $50 \%$ of the contacted bubbles coalesce. Compared with bubble swarm experiments, the relative uncertainties decrease due to the relatively stable environment. However, the bubble pairs are simplified and labelled as "coalescence” or "non-coalescence”. Therefore, important information representing the efficiency of inhabitation, such as bubble coalescence time or lifetime of liquid film between bubbles, is still missing.

The film balance technique, unlike the previous techniques, can provide important information to understand the bubble coalescence process. For example, both the drainage time and thickness of liquid films are taken in account. However, the exposure of the liquid film to air results in many uncertainties, such as the contamination, evaporation, and thermal or mechanical fluctuations from the environment. Since no real bubble is generated, the film balance technique still cannot provide important information such as bubble deformation and post-coalescence oscillation. ${ }^{14}$

This communication demonstrates that microfluidics is a powerful tool to understand the complex bubble dynamics. The design of the experiments in the micro-channels eliminates the effects of longrange inertial forces, therefore allows for the investigation of only short-range effects (e.g. colloidal forces) on the bubble coalescence process. This tool also circumvents many of the above-mentioned limitations. For example, the laminar flow behaviour allows the generation of extremely wellcontrolled bubble size. ${ }^{15,16}$ Furthermore, the confinement of a microchannel guarantees the wellcontrolled transport of microbubbles. Therefore, microfluidic devices have been utilised to study bubble coalescence behaviours. ${ }^{17,} 18$ However, studies on bubble coalescence in microfluidic devices were limited mainly to the effect of hydrodynamics. We aim to report a different perspective where the CCC of salt solutions is determined using a microfluidic flow-focusing device. Controlling bubble generation and coalescence in a microfluidic device allows a more reliable and precise quantitative determination of CCC as compared to existing conventional techniques. 


\section{EXPERIMENTS}

Air bubbles were generated in aqueous sodium halide solutions ( $\mathrm{NaF}, \mathrm{NaCl}, \mathrm{NaBr}$ and $\mathrm{NaI}$ ) using the flow-focusing geometry, Fig. 1. The flow rates of salt solution and air were $500 \mu \mathrm{L} / \mathrm{h}$ and 100 $\mu \mathrm{L} / \mathrm{h}$, respectively. The frequency of bubble generation was about $52 \pm 5 \mathrm{~Hz}$. Coalescence of air bubbles was observed at the expansion of the microfluidic channels, Fig. 1 (a). Standard soft lithography technique was used to fabricate microfluidic device in poly-dimethylsiloxane (PDMS). The PDMS device was bonded to a glass slide using air plasma. The width of the main channels and the orifice are $100 \mu \mathrm{m}$ and $50 \mu \mathrm{m}$, respectively. Fig. 1(a) shows the detailed geometry of the microchannels. The micro-channels have a height of about $30 \mu \mathrm{m}$. Fig. 1(b) shows a representative snapshot of the bubble generation process at the flow-focusing junction. The subsequent bubble coalescence occurs at the expansion, Fig. 1(c). A high-speed camera (Miro 3, Vision Research) mounted on an inverted microscope (Nikon Ti-E, Japan) recorded the bubble images.

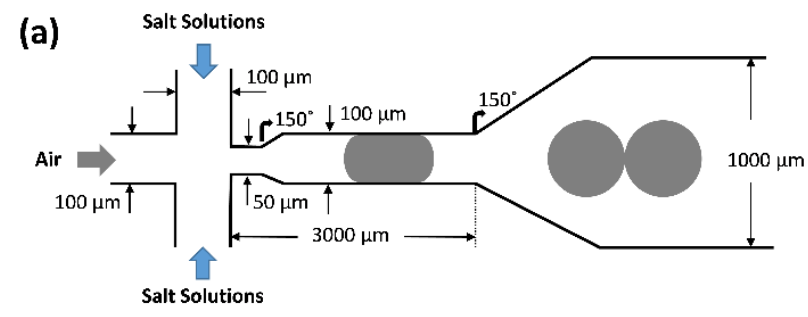

(b)

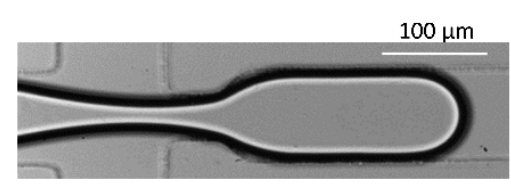

(c)

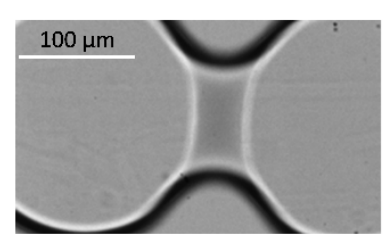

Fig. 1 (a) Schematic diagram of the flow-focusing microfluidic device; (b) Image of the bubble generation and (c) coalescence in $0.5 \mathrm{M} \mathrm{NaCl}$ solution with a total $\mathrm{NaCl}$ solution flow rate of $500 \mu \mathrm{L} / \mathrm{h}$ and an air flow rate $100 \mu \mathrm{L} / \mathrm{h}$. The depth of the channel is about $30 \mu \mathrm{m}$. The scale bar indicated in the figure represent $100 \mu \mathrm{m}$. 
Sequential layer-by-layer deposition of polyelectrolytes (poly (allylamine hydrochloride) i.e. PAH (98\% Sigma-Aldrich, USA) and poly (sodium 4-styrenesulfonate) i.e. PSS (98\% Sigma-Aldrich, USA)) was used to yield hydrophilic micro-channels in the PDMS-based microfluidic devices. ${ }^{19}$ Briefly, the first positively charged PAH layer was deposited onto negatively charged PDMS surface treated by air plasma. Then the negatively charged PSS layer was deposited onto the PAH layer. After 8 deposition cycles, a (PAH/PSS) 8 polyelectrolyte multilayer was coated on the channel wall. The final washing step was performed with deionized water to remove traces of contaminations in the micro-channels.

Deionized (DI) water was used to make the salt solutions, i.e. solutions of NaF (99\% SigmaAldrich, USA), $\mathrm{NaCl}$ (99.5 \% Sigma-Aldrich, USA), NaBr (99\% Sigma-Aldrich, Israel) and NaI (99\% Merck, Germany). Air and salt solutions were loaded in gas tight glass syringes and pumped into the micro-channels using a syringe pump (SPM 100 S-FLUIDPUMP, Singapore). Videos of bubble coalescence were recorded at a frame rate of 13,000 frames per second (fps) and processed via ImageJ (V1.48, NIH, USA). The coalescence time was calculated as the time interval from the touching of two air bubble interfaces to the $1^{\text {st }}$ frame of bubble coalescence. The uncertainty for the measurement of coalescence time is the interval between two frames, i.e. $76.92 \mu$ s. Four to ten measurements were taken to obtain the average coalescence time for each solution. Fig. 2 shows image sequences of the coalescence process of two bubbles in a $0.75 \mathrm{M} \mathrm{NaF}$ solution. We identified four consecutive stages of the coalescence process: approaching (Fig. 2a), touching (Fig. 2b), liquid film draining (Fig. 2c) and coalescence (Fig. 2d). The average diameter and the collision speed of the bubbles were $255 \pm 4 \mu \mathrm{m}$ and $0.022 \pm 0.004 \mathrm{~m} / \mathrm{s}$, respectively. The bubble size in this study is at least one order of magnitude smaller than the one measured in all existing conventional techniques. ${ }^{2}$ The bubble approaching speed in this study is in the "instant coalescence" regime $(0.0012-0.14 \mathrm{~m} / \mathrm{s})$, i.e. bubble coalescence time is smaller than $0.01 \mathrm{~s}$, for the bubble stability in water. ${ }^{20}$ Our results with all the bubble coalescence times smaller than $0.001 \mathrm{~s}$ validate the existence of this regime. 
(a)

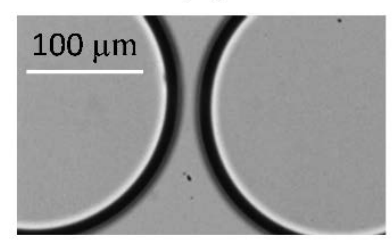

(c)

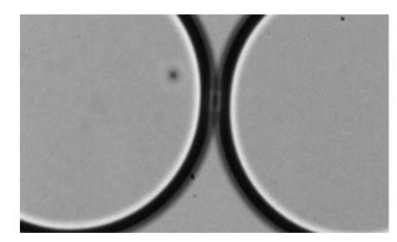

(b)

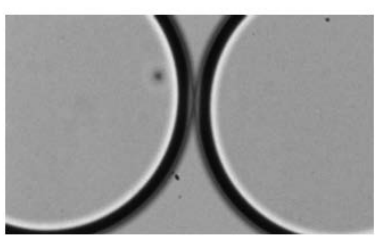

(d)

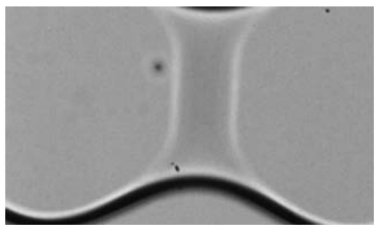

Fig. 2 Image sequences of air bubble coalescence in $0.75 \mathrm{M} \mathrm{NaF}$ solution in different stages: (a) approaching; (b) touching ( $\left.t=t_{0}\right)$; (c) liquid film draining and (d) $1^{\text {st }}$ frame of coalescing $\left(t=t_{\text {crit }}\right.$ ). The scale bar indicated in the figure represent $100 \mu \mathrm{m}$.

\section{RESULTS AND DISCUSSION}

Fig. 3a shows the coalescence time for colliding bubble pairs in sodium halide solutions of different concentrations up to $0.5 \mathrm{M}$. A critical salt concentration exists for the shortest bubble coalescence time. At this salt concentration, bubble coalescence occurs most efficiently. The salt concentrations are referred to as the critical coalescence concentration (CCC) as bubble dynamics moves from coalescence to inhibition. The CCCs are found as $0.070 \pm 0.010 \mathrm{M}, 0.0850 \pm 0.010 \mathrm{M}, 0.175 \pm 0.025$ $\mathrm{M}$ and $0.325 \pm 0.025 \mathrm{M}$ for $\mathrm{NaF}, \mathrm{NaCl}, \mathrm{NaBr}$ and $\mathrm{NaI}$, respectively. It is important to note that our measured results of CCC are within the range of previously published results that were determined via the film balance technique, i.e. $0.080 \mathrm{M}, 0.115 \mathrm{M}, 0.195 \mathrm{M}$ and $0.345 \mathrm{M}$ for $\mathrm{NaF}, \mathrm{NaCl}, \mathrm{NaBr}$ and NaI, respectively. ${ }^{21,22}$ However, the uncertainty of bubble coalescence time using our microfluidic method $(<6 \%)$ is much smaller than that of the film balance technique (as high as $20 \%$ ). ${ }^{22}$ Despite of the consistency of the critical concentration measurements, the bubble coalescence times (range from $100-500 \mu \mathrm{s}$ ) that we measured in the microfluidic device is much shorter than the reported values (0.1 - 30 s) measured with liquid film balance technique., ${ }^{22}$ This discrepancy is due to the difference in the interface approach speed. The approach speed in this study $(22 \mathrm{~mm} / \mathrm{s})$ is much greater than the one that drives film drainage in film balance technique $(10-300 \mu \mathrm{m} / \mathrm{s})$. It has been reported that there exists a critical approach speed (around $35 \mu \mathrm{m} / \mathrm{s}$ ) above which the bubbles coalesce instantly ( $<0.1 \mathrm{~s}$ ), whereas the critical concentration of salts is independent of the approach speed. ${ }^{22}$ Our results show the validation of the proposed microfluidic method. Furthermore, the time scale of the bubble coalescence in our study, i.e. hundreds of microseconds, is more relevant to the real systems like bubble columns and flotation separation where the bubble contact time in solutions is shorter than $1 \mathrm{~s}$, as compared with the time scale (as long as $20 \mathrm{~s}$ ) of the film balance experiments. ${ }^{22}$ The laminar flow in micro- 
channels eliminates the uncertainties of bubble coalescence due to the turbulence in bubble swarm experiments. The microfluidic method also provides a reliable and precise bubble coalescence time rather than qualitative description as "coalescence” or "non-coalescence” in the bubble pair method.

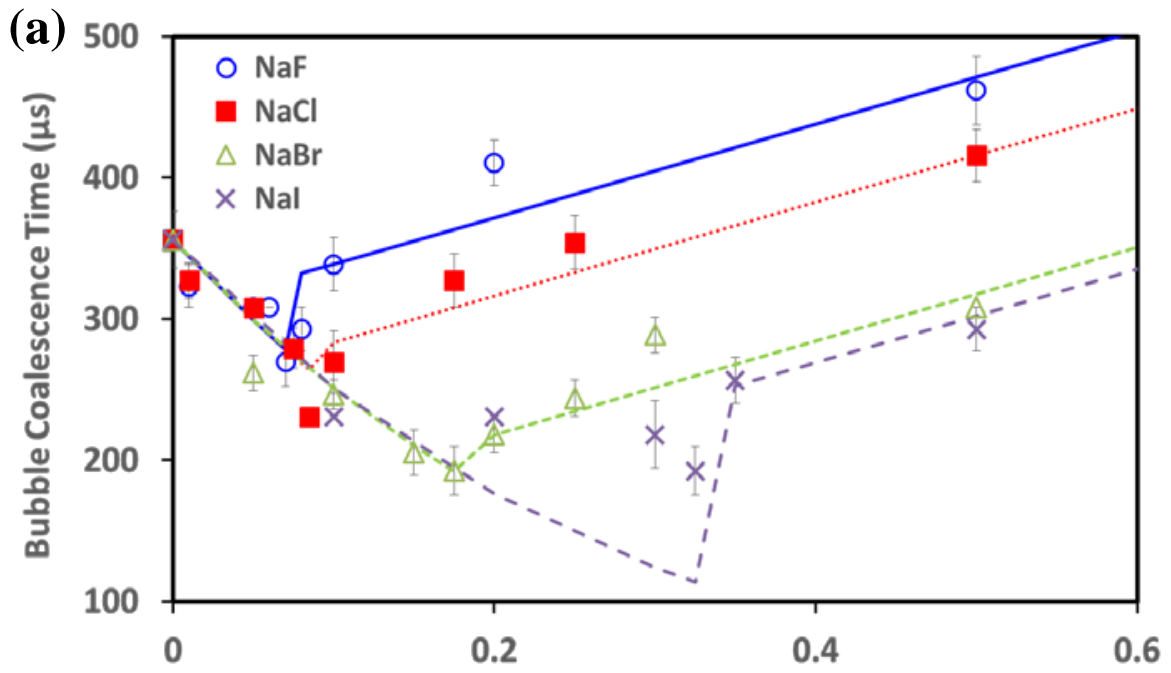

(b)

Salt Concentration (M)

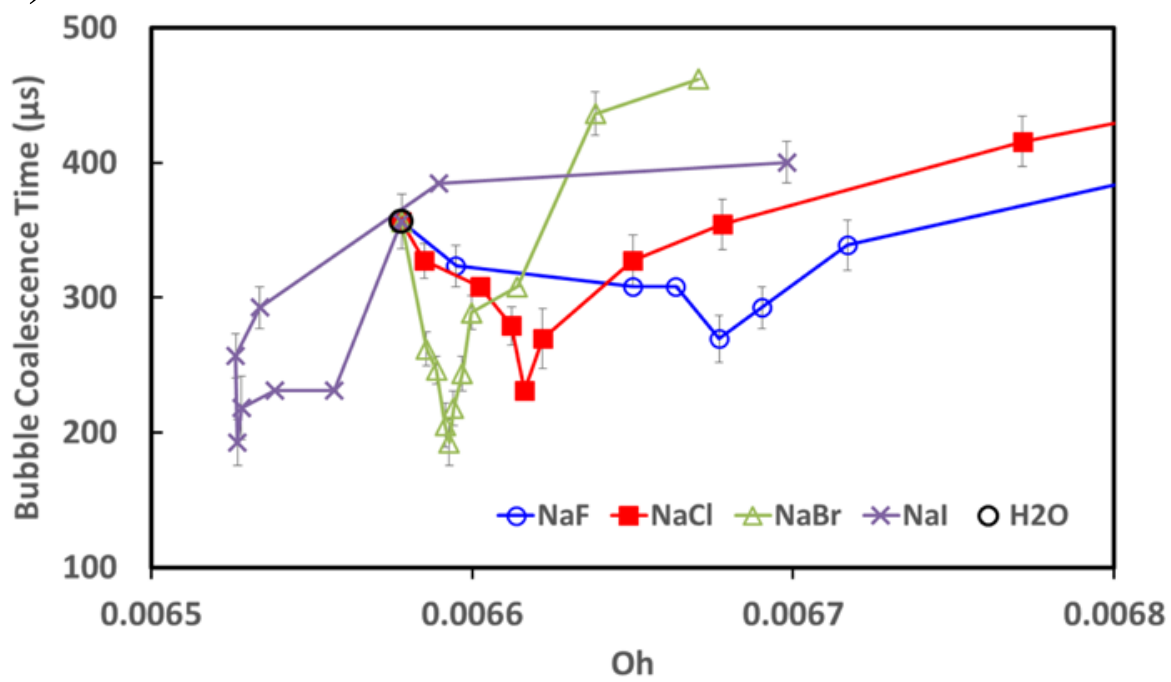

Fig. 3 Bubble coalescence time versus different salt concentrations (Fig. 3a) and Ohnesorge number (Fig. 3b). The symbols represent the different types of salts: $\mathrm{NaF}, \mathrm{NaCl}, \mathrm{NaBr}$ and $\mathrm{NaI}$. The lines in Fig. 3a represent the simulation results via Eqs. (1) - (2). The lines in Fig. 3b guide the eyes only. The error bars represent the standard error $(n=4-10)$. The critical coalescence concentration refers to the shortest coalescence time as indicated via arrows.

Fig. 3a shows that the obtained CCCs for the monovalent anions are not the same, but increase with the anion radius. The ion-specific effect on bubble coalescence in sodium halide salt solutions follows the order of inhibition capability: $\mathrm{NaF}>\mathrm{NaCl}>\mathrm{NaBr}>\mathrm{NaI}$. At concentration smaller than CCC, the curves for bubble coalescence time almost overlap. However, at concentrations higher than CCC, the 
curves deviate from one another significantly. The relationship between bubble coalescence time and salt concentration in Fig.3 can be quantified via the following fitting functions:

$t=t_{0} \exp (-C / 0.284) \quad t \leq t_{\text {Crit }}$

$t=A+331.2 \times C \quad t>t_{\text {Crit }}$

(2)

where $t$ is the average bubble coalescence time at the salt concentration $C(0-0.6 \mathrm{M}), t_{\text {crit }}$ is the bubble coalescence time at the critical salt concentration, $t_{0}$ is the average bubble coalescence time (356.12 $\mu \mathrm{s})$ in pure water and $A$ is a constant which decreases with the anion radius: $A_{N a F}(305.68)>$ $A_{N a C l}(250.20)>A_{N a B r}(151.93)>A_{N a I}$ (136.60). In the regimes of low salt concentration as shown in Eq. (1), the bubble coalescence is controlled by the repulsion between the electrical double layers at the bubble surfaces, which becomes weak with increasing salt concentration due to the compression of the double layers. The inhibition of bubble coalescence at high salt concentration is still not well understood. 23, 24 However, Eq. (2) reveals that at high salt concentration the bubble coalescence time may increase with salt concentration with the same slope despite of the ion-specific effect on the constant $A$. Gibbs elasticity of the liquid film caused by the surface tension gradient, and hydrophobic forces between bubble interfaces due to dissolved gases in salt solutions are believed to be related to the ion-specific effect on bubble coalescence inhibition at high salt concentration. ${ }^{2}$ Nonetheless, the coalescence time curves are similar to the change in surface tension of salt solutions, which is referred to as the Jones-Ray effect. ${ }^{25}$

We also plot the coalescence time against Ohnesorge number (Oh $=\mu / \sqrt{\rho \sigma D}$ where $\mu, \rho, \sigma$, and $D$ are viscosity, density, surface tension and bubble diameter, respectively), which is the ratio of viscous forces to inertial and surface tension forces, in Fig. 3b. It has been reported that the coalescence of bubbles and drops in an outer fluid is related to this dimensionless number and a phase diagram is created to identify different regimes of the coalescence process. ${ }^{26}$ However, Fig. 3b shows that the change of Ohnesorge number in current study is very small $(0.0065-0.0068)$, therefore we can safely neglect the effects of physical properties of salt solutions on the bubble coalescence process.

Nearly 130 years on since the ion-specific effect on protein precipitation had been found by Hofmeister ${ }^{27}$, its satisfactory explanation is still a challenge of the classic theories, including the Debye-Hückel and DLVO theories. Only phenomenological set of rules, such as the law of matching water affinities ${ }^{28}$ and combining rules of ions to inhibit bubble coalescence, ${ }^{11}$ are successfully applied to explain the effect. Nevertheless, microfluidics proves to be a useful tool for investigating the ionspecific effect on bubble coalescence. 


\section{CONCLUSIONS}

In summary, we report a microfluidic method to investigate the ion-specific effect on bubble coalescence. We directly visualised the bubble coalescence in a micro-channel using high-speed imaging and evaluated the bubble coalescence time to determine the critical coalescence concentration of sodium halide solutions. We found the capacity of sodium halide salts to inhibit bubble coalescence follows the order of $\mathrm{NaF}>\mathrm{NaCl}>\mathrm{NaBr}>\mathrm{NaI}$. Compared with conventional techniques, the microfluidic method overcomes their inherent uncertainties and provides a useful tool for the future studies on ion-specific effect.

\section{AUTHOR INFORMATION}

Corresponding Author

*E-mail: nam-trung.nguyen@griffith.edu.au

\section{Notes}

The authors declare no competing finacial intersts.

\section{ACKNOWLEDGEMENTS}

The authors acknowledge the Australia Research Council (ARC) for financial support (project number DP140101089). S. H. Tan gratefully acknowledges the support of the Griffith University Post-doctoral fellowship, New Researcher grant, ARC linkage grant (LP150100153) and Griffith University-Peking University collaboration grant.

\section{REFERENCES}

1. Nguyen, A. V.; Schulze, H. J. Colloidal science of flotation; Marcel Dekker: New York, 2004. p 840.

2. Firouzi, M.; Howes, T.; Nguyen, A. V. A quantitative review of the transition salt concentration for inhibiting bubble coalescence. Advances in Colloid and Interface Science 2015, 222, 305-318.

3. Marrucci, G.; Nicodemo, L. Coalescence of gas bubbles in aqueous solutions of inorganic electrolytes. Chemical Engineering Science 1967, 22 (9), 1257-1265.

4. Prince, M. J.; Blanch, H. W. Transition electrolyte concentrations for bubble coalescence. AlChE Journal 1990, 36 (9), 1425-1429.

5. Lessard, R. R.; Zieminski, S. A. Bubble Coalescence and Gas Transfer in Aqueous Electrolytic Solutions. Industrial \& Engineering Chemistry Fundamentals 1971, 10 (2), 260-269.

6. Cho, Y. S.; Laskowski, J. S. Bubble coalescence and its effect on dynamic foam stability. The Canadian Journal of Chemical Engineering 2002, 80 (2), 299-305. 
7. Laskowski, J. S.; Thone, T.; Williams, P.; Ding, K. Fundamental properties of the polyoxypropylene alkyl ether flotation frothers. International Journal of Mineral Processing 2003, 72 (1-4), 289-299.

8. Grau, R. A.; Laskowski, J. S. Role of Frothers in Bubble Generation and Coalescence in a Mechanical Flotation Cell. The Canadian Journal of Chemical Engineering 2006, 84 (2), 170-182.

9. Kracht, W.; Finch, J. A. Bubble break-up and the role of frother and salt. International Journal of Mineral Processing 2009, 92 (3-4), 153-161.

10. Nassif, M.; Finch, J. A.; Waters, K. E. Developing critical coalescence concentration curves for industrial process waters using dilution. Minerals Engineering 2013, 50-51, 64-68.

11. Craig, V. S. J.; Ninham, B. W.; Pashley, R. M. The effect of electrolytes on bubble coalescence in water. Journal of Physical Chemistry 1993, 97 (39), 10192-7.

12. Quinn, J. J.; Sovechles, J. M.; Finch, J. A.; Waters, K. E. Critical coalescence concentration of inorganic salt solutions. Minerals Engineering 2014, 58, 1-6.

13. Kracht, W.; Rebolledo, H. Study of the local critical coalescence concentration (I-CCC) of alcohols and salts at bubble formation in two-phase systems. Minerals Engineering 2013, 50-51 (0), 77-82.

14. Bournival, G.; Ata, S.; Karakashev, S. I.; Jameson, G. J. An investigation of bubble coalescence and post-rupture oscillation in non-ionic surfactant solutions using high-speed cinematography. Journal of Colloid and Interface Science 2014, 414, 50-58.

15. Nguyen, N.-T.; Wereley, S. T. Fundamentals and Applications of Microfluidics; Artech House: Boston London, 2002.

16. Chong, Z. Z.; Tor, S. B.; Loh, N. H.; Wong, T. N.; Ganan-Calvo, A. M.; Tan, S. H.; Nguyen, N.-T. Acoustofluidic control of bubble size in microfluidic flow-focusing configuration. Lab on a Chip 2015, 15 (4), 996-999.

17. Yang, L.; Wang, K.; Tan, J.; Lu, Y.; Luo, G. Experimental study of microbubble coalescence in a T-junction microfluidic device. Microfluidics and Nanofluidics 2012, 12 (5), 715-722.

18. Wu, Y.; Fu, T.; Zhu, C.; Ma, Y.; Li, H. Z. Bubble coalescence at a microfluidic T-junction convergence: from colliding to squeezing. Microfluidics and Nanofluidics 2014, 16 (1), 275-286.

19. Bauer, W.-A. C.; Fischlechner, M.; Abell, C.; Huck, W. T. S. Hydrophilic PDMS microchannels for high-throughput formation of oil-in-water microdroplets and water-in-oil-in-water double emulsions. Lab on a Chip 2010, 10 (14), 1814-1819.

20. Del Castillo, L. A.; Ohnishi, S.; Horn, R. G. Inhibition of bubble coalescence: Effects of salt concentration and speed of approach. Journal of Colloid and Interface Science 2011, 356 (1), 316-324. 21. Firouzi, M. Drainage and Stability of Foam Films during Bubble Coalescence in Aqueous Salt Solutions. Doctorate Degree, University of Queensland2014.

22. Firouzi, M.; Nguyen, A. V. Effects of monovalent anions and cations on drainage and lifetime of foam films at different interface approach speeds. Advanced Powder Technology 2014, 25 (4), $1212-1219$.

23. Nguyen, P. T.; Hampton, M. A.; Nguyen, A. V.; Birkett, G. R. The influence of gas velocity, salt type and concentration on transition concentration for bubble coalescence inhibition and gas holdup. Chemical Engineering Research and Design 2012, 90 (1), 33-39.

24. Henry, C. L.; Craig, V. S. J. The link between ion specific bubble coalescence and Hofmeister effects is the partinioning of ions within the interface. Langmuir 2010, 26 (9), 6478-6483.

25. Nguyen, K. T.; Nguyen, A. V.; Evans, G. M. Interactions between halide anions and interfacial water molecules in relation to the Jones-Ray effect. Physical Chemistry Chemical Physics 2014, 16 (45), 24661-24665.

26. Paulsen, J. D.; Carmigniani, R.; Kannan, A.; Burton, J. C.; Nagel, S. R. Coalescence of bubbles and drops in an outer fluid. Nature Communications 2014, 5, 3182.

27. Hofmeister, F. Zur Lehre von der Wirkung der Salze. Archiv für experimentelle Pathologie und Pharmakologie 1888, 24 (4), 247-260. 
28. Collins, K. D. Charge density-dependent strength of hydration and biological structure. Biophysical Journal 1997, 72 (1), 65-76.

TOC

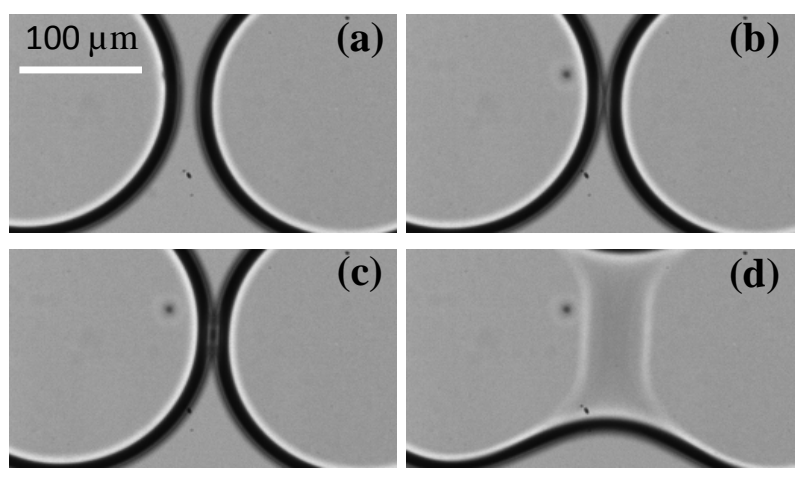

